\title{
Concepts for modeling impacts without friction
}

\author{
Journal Article
}

Author(s):

Glocker, C.

Publication date:

2004-03

Permanent link:

https://doi.org/10.3929/ethz-b-000050867

Rights / license:

In Copyright - Non-Commercial Use Permitted

Originally published in:

Acta Mechanica 168(1-2), https://doi.org/10.1007/s00707-004-0076-3 


\title{
Concepts for modeling impacts without friction
}

\author{
Ch. Glocker, Zurich, Switzerland \\ Received August 8, 2003; revised November 7, 2003 \\ Published online: March 2, 2004 ㄷ Springer-Verlag 2004
}

\begin{abstract}
Summary. There are three basic equations in mechanics for treating collisions: the law of impact, kinematic compatibility, and energetic consistency. In this paper, the conditions are examined under which a natural extension of the dynamics at an impact is possible without taking additional impact laws, and which additional assumptions have to be made to solve the impact for different classes of systems. It will be shown that Newton's law of impact for two colliding point masses can be derived from the concept of energy conservation and the principle of maximum dissipation, and has therefore not to be regarded as an independent equation. Moreover, it can be assigned to single-contact impacts in multibody systems as soon as the classical definition of perfect constraints is being extended to impulsive dynamics and unilateral contacts. It will further be shown that the principle of maximum dissipation leads to a unique postimpact velocity in the case of multi-contact collisions. In all other cases, however, the velocities remain undetermined, and laws of impact have to be postulated as additional and independent equations, whereas the classic definition of the restitution coefficient as a dissipation parameter can still be kept.
\end{abstract}

\section{Introduction}

It has been known since Galileo Galilei (Discorsi, The Sixth Day) [1] that impact forces can become unlimited. Huygens had been examining completely elastic collisions between two point masses since 1656. He recognized the fact, that besides conservation of momentum and kinetic energy the relative motion of two bodies has to be taken into account in order to be able to formulate a universally valid law of impact. His law $v-V=C-c$, describing the relative velocities inversion during the elastic impact, is extended by Newton in 1687 by the restitution coefficient $\varepsilon$ in order to accommodate possible losses of energy during the collision. The form $\varepsilon(v-V)=(C-c)$ serves Newton as an experimental confirmation of his third law "actio $=$ reactio" [1]. By setting $\varepsilon=1$ Huygens impact law for the elastic case is obtained, whereas $\varepsilon=0$ describes the limiting case of maximum dissipation possible, such that the bodies do not penetrate after impact but keep moving with a common velocity. The restitution coefficient $\varepsilon$ is regarded as a measure of dissipated energy during the impact in this context.

For systems composed of several elastic and rigid bodies, conservation of linear and angular momentum does not lead to the target aimed at. Instead, the Newton-Euler equations have to be established for each body in order to obtain sufficient equations describing the dynamics. If one allows these bodies to have impacts at several contact points, one restitution coefficient is not enough any more to unambiguously determine the post-impact velocities of all degrees of freedom, since it is not known how the kinetic energy is distributed among the single bodies. One normally postulates ad-hoc impact laws that are more or less suited to describe reality in all cases. Known problems consist either in obtaining too many possible post-impact velocities 
while using the restitution coefficients provided by the impact laws, leading to energetic or kinematic inconsistency, or in not being able to reach certain velocities at all. In the latter case, behavior observed in experiments can sometimes not be reproduced in calculations by any choice of the impact parameters in use.

Another difficulty consists in a widely spread misunderstanding of the restitution coefficients. They are material-pairing-constants only in the fewest cases, but have to be generally understood as a measure of dissipation concerning the chosen spatial discretization level of the mechanical system. This is addressed by many people in saying that the restitution coefficients depend in some way on the geometry of the colliding bodies, meaning that they must somehow take into account the wave propagation process initiated by the collision. Since dissipation in mechanical systems has also to be understood as a transfer of energy to mechanical degrees of freedom which are not contained in the mathematical model, the restitution coefficients can be diminished being associated with a refinement of the discretization. In order to obtain the right dynamic behavior for the macroscopic degrees of freedom, the impact can finally be seen as completely inelastic when using a continuum model.

One reason for the mentioned difficulties in setting up the impact laws for multi-pointcollisions is the fact, that it has not yet been examined from which point and to what extend a formulated impact law may be understood as an independent equation, in order to avoid contradictions with the basic equations of kinetics and the kinematic and energetic restrictions. This question is the main topic of this article. It will not yet attempt obtaining a complete parametrization free of contradictions in form of a general impact law, but propose an appropriate setting for a theoretical framework in which any impact law should reside. Only the impact itself will be examined. Pre- and post-impact motions will not be discussed. As an impact we will understand a velocity jump which occurs at a discrete point in time, and which is associated with infinite impulsive forces as a consequence of finite, non-disappearing masses in the system. Processes with rapidly changing velocities without discontinuities will not be understood as impacts, as e.g., models containing local stiffness in the contact points of the colliding bodies. Only collisions will be investigated. Impulsive forces applied from outside that can be regarded as external impact excitation, e.g. the impact from the queue on the ball when playing billiard, will not be examined in this context. All discussions will be limited to scleronomic systems. Even though explicit time-dependencies do not represent a burden they will not be treated in favor of clearness. Influences of Coulomb friction during the collision will not be permitted as well. The concept of perfect constraints is essential in the sense of a general and structural procedure according to classic mechanics. Coulomb friction would destroy it. Wave effects during the collision, however, are explicitly permitted.

The structure of this article is being lead by the philosophy of going from basic to more sophisticated statements, and not introducing impact laws as independent equations as long as a natural generalization of the dynamics at the impact is possible by using only the basic equations of kinematics, dynamics and energy. In order to display the gained statements in a most descriptive manner a geometric approach, based on the kinetic metric, will be chosen. Section 2 is about Newton's impact problem, the collision of two point masses. It is being treated by the help of conservation of linear momentum and two energetic limiting cases, namely conservation of kinetic energy and the principle of maximum dissipation. This leads to the classical law of impact, which therefore does not need to be introduced as an independent equation. In Sect. 3, the example of two colliding beams shows the restitution coefficient's dependency of the spatial dicretization level. In a first step both beams will be modelled as onedimensional elastic continua. The collision will naturally be assumed to be completely inelastic. In a second step the beams are assumed to be rigid, and the impact is solved by the equations 
from Sect. 2. The beams' average velocities match only, if the restitution coefficient for the rigid body model is being chosen according to the ratio of the beams' lengths. Section 4 deals with the single-contact impact in a multibody system. By using a collision model based on perfect unilateral constraints, it can be shown that the impact dynamics is still obtained in a natural way, and that the impact law does not yet need to be interpreted as an independent equation, just as in Sect. 2. The limiting cases of the completely elastic and inelastic impact can be geometrically interpreted as a reflection and orthogonal projection, and the impact itself as a necessary action to keep a curve on a manifold when their boundary is reached under a nonvanishing angle. In Sect. 5, multi-contact collisions are addressed. While the principle of maximum dissipation still yields a unique extension of the dynamics at the impact, the postimpact velocities for any other level of dissipation may be underdetermined. The different dissipation levels lead to a foliation of the energy ball into spheres, such that the classical concept of using a dissipation coefficient to characterize the energetic property of an impact can still be kept. However, additional equations, the impact laws are needed to locate the impact event on the according spheres and to make the whole impact well-defined. Finally, Sect. 6 shows one concept that is used today to treat the multi-contact case of Sect. 5. It is based on the orthogonal decomposition of the pre-impact velocity with respect to a pair of convex cones and admits a very clear geometric interpretation of the impact process. It does, however, not address every impact event possible, but only a sub-class that we call impacts with global dissipation index. We will discuss the mechanical meaning of this class of impacts by examples and and give a representation of the impact law in local contact coordinates.

\section{Collision of two bodies}

We consider a mechanical system of two interacting bodies defined on $\mathbb{R} \times \mathbb{R}$. The masses of the two bodies are $m_{1}$ and $m_{2}$, and the velocities of their centers of mass $v_{1}$ and $v_{2}$. The interaction force between the two bodies is denoted by $\Lambda$. This configuration was also used by Newton to experimentally confirm his third law "actio = reactio" [1]. The experimental setup is shown in Fig. 1. Newton's impact law must not be considered as an independent physical principle, but can be derived from conservation principles and energetic considerations.

Let $\left(v_{1}^{-}, v_{2}^{-}\right)$be given velocities of the centers of mass at time $t^{-}$, and $\left(v_{1}^{+}, v_{2}^{+}\right)$unknown velocities at time $t^{+}\left(t^{+} \geq t^{-}\right)$. Further, we consider the impulse $p$ and the kinetic energy $T$ of the system,

$p=m_{1} v_{1}+m_{2} v_{2}, \quad T=\frac{1}{2} m_{1} v_{1}^{2}+\frac{1}{2} m_{2} v_{2}^{2}$.
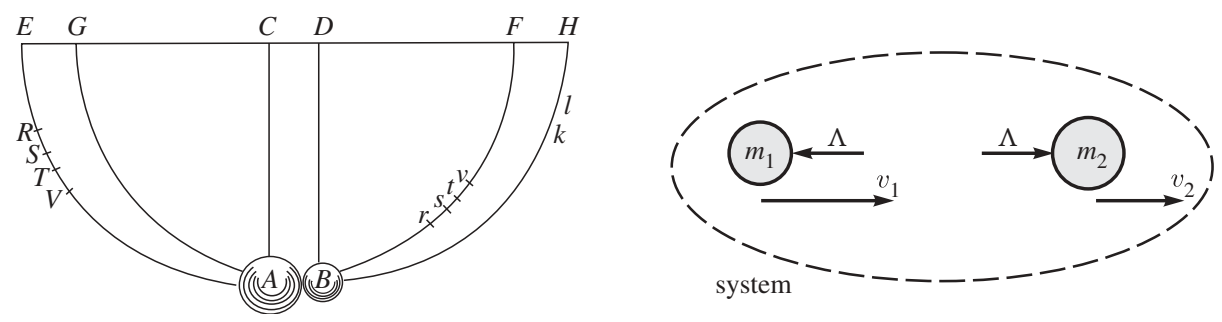

Fig. 1. Collision of two bodies 
A regular transformation of the velocities $\left(v_{1}, v_{2}\right) \rightarrow(u, \gamma)$,

$\left(m_{1}+m_{2}\right) u:=m_{1} v_{1}+m_{2} v_{2}, \quad \gamma:=v_{2}-v_{1}$

leads to

$v_{1}=u-\frac{m_{2}}{m_{1}+m_{2}} \gamma, \quad v_{2}=u+\frac{m_{1}}{m_{1}+m_{2}} \gamma$.

Equation (1) can now be written as

$p=\left(m_{1}+m_{2}\right) u, \quad T=\frac{1}{2}\left(m_{1}+m_{2}\right) u^{2}+\frac{1}{2} \frac{m_{1} m_{2}}{m_{1}+m_{2}} \gamma^{2}$,

where $u$ is the velocity of the center of mass of the overall system and $\gamma$ the relative velocity of $m_{2}$ with respect to $m_{1}$.

The impulse of the system is conserved, because there are no external forces acting. This leads with Eq. (4) to

$p^{+} \equiv p^{-} \quad \Rightarrow \quad\left(m_{1}+m_{2}\right) u^{+} \equiv\left(m_{1}+m_{2}\right) u^{-} \quad \Rightarrow \quad u^{+} \equiv u^{-}$.

Therefore, the center of mass of the whole system is not accelerated. This result is valid for arbitrary force interactions $\Lambda$ between the bodies and arbitrary times $t^{-}, t^{+}$. We are free to chose a spring-damper element, an instantaneous impulsive force, or a continuum model that takes into account the elasticity of the bodies; the nature of the interaction model between the two bodies has no influence on the achieved results. From Eqs. (5) and (3) we get the difference in the velocities

$v_{1}^{+}-v_{1}^{-}=-\frac{m_{2}}{m_{1}+m_{2}}\left(\gamma^{+}-\gamma^{-}\right), \quad v_{2}^{+}-v_{2}^{-}=+\frac{m_{1}}{m_{1}+m_{2}}\left(\gamma^{+}-\gamma^{-}\right)$

and their quotient as

$\frac{v_{1}^{+}-v_{1}^{-}}{v_{2}^{+}-v_{2}^{-}}=-\frac{m_{2}}{m_{1}}$.

This ratio is already unique, while the differences themselves depend still on the relative velocity $\gamma$. The unknown velocity difference in (6) may further be specified by using energetic considerations: With the equality $u^{+}=u^{-}$from (5) we obtain by (4) the expression

$T^{+}-T^{-}=\frac{1}{2} \frac{m_{1} m_{2}}{m_{1}+m_{2}}\left(\gamma^{+2}-\gamma^{-2}\right)$.

This difference in kinetic energy depends on the chosen interaction model. For example, if there is a relative acceleration between $m_{1}$ and $m_{2}$ provided by a motor or a prestressed spring, the kinetic energy $T^{+}$will be larger than $T^{-}$. By modelling the interaction using a passive springdamper element, the kinetic energy $T^{+}$becomes zero for $t^{+} \rightarrow \infty$. Therefore, the difference in kinetic energy does not necessarily show negative values and indicate dissipation, but may also be positive. Note also that the time interval $\left[t^{-}, t^{+}\right]$is not yet constricted and can still have arbitrary length.

Let us now discuss the behavior of the system when a collision takes place. We allow for the force interactions $\Lambda$ values unequal to zero only in the case of contact between the bodies. Otherwise, if the bodies are separated, we demand $\Lambda=0$. With this assumption we may chose $t^{+}$and $t^{-}$such that there is no force interaction outside the interval $\left[t^{-}, t^{+}\right]\left(t^{+}=+\infty\right.$ is allowed). A collision requires the two bodies to move together. Thus, the relative velocity at the start of the collision $t^{-}$must be negative, $\gamma^{-}<0$. On the other hand, the relative velocity at $t^{+}$ must be positive, $\gamma^{+} \geq 0$. Otherwise, the bodies would interpenetrate each other due to the missing force interactions outside of $\left[t^{-}, t^{+}\right]$that we have demanded in our previous assump- 
tion. As a consequence from $\left(\gamma^{-}<0, \gamma^{+} \geq 0\right)$, the interaction force has to act in mean as a compressive magnitude during the collision time interval, $\int_{\left[t^{-}, t^{+}\right]} d \Lambda=\Lambda>0$. We further assume the collision to be dissipative with respect to the chosen discretization level with two degrees of freedom, $T^{+}-T^{-} \leq 0$, in order to later restrict the dissipation coefficient $\varepsilon$ to nonnegative values not exceeding 1 . This assumption excludes on the one hand any active behavior of the collision partners as in a pinball machine, and on the other hand any energy transfer from microscopic internal degrees of freedom to our macroscopic discretization level. The latter is guaranteed, for example, if eventual internal oscillations of both collision partners have been faded away at the time of collision, i.e., if the value of $T^{-}$is invariant under any spatial discretizazion of the system, including any possible continuum model.

By the previous assumptions, we may now consider two limiting cases, for which the relative velocity after the collision $\gamma^{+}$can be calculated:

Conservation of kinetic energy: Equation (8) leads together with $\gamma^{-}<0, \gamma^{+} \geq 0$ to

$T^{+}=T^{-} \quad \Rightarrow \quad \gamma^{+}=-\gamma^{-}$.

The relative velocity is inverted by the collision, and one speaks about a completely elastic collision behavior.

Maximum dissipation: This case is conveniently stated as an optimization problem on (8): For given $\gamma^{-}$, find $\gamma^{+}$such that

Minimize $T^{+}-T^{-}$under $\gamma^{+} \geq 0 \Rightarrow \gamma^{+}=0$.

Here, the two bodies move with a common velocity after the collision, and the collision itself is called completely inelastic.

In order to describe dissipation levels between the two limit cases, a restitution coefficient $\varepsilon$ is introduced,

$\gamma^{+}=-\varepsilon \gamma^{-} \quad(0 \leq \varepsilon \leq 1)$.

This equation is known as Newton's impact law. It covers the limit cases of a complete elastic $(\varepsilon=1)$ and a complete inelastic $(\varepsilon=0)$ collision behavior. The restitution coefficient $\varepsilon$ serves as a measure for the amount of dissipation at the collision.

Considering the two bodies as rigid, the change in the relative velocity $\gamma^{-}$to $\gamma^{+}$has to be instantaneous to prevent a penetration of the two bodies. This causes the time interval of the collision to shrink to one point, $\left[t^{-}, t^{+}\right]=\left\{t^{-}=t^{+}=: t^{\star}\right\}$, and the interaction between the two bodies to become impulsive, $\int_{\left\{t^{*}\right\}} \mathrm{d} \Lambda=\Lambda>0$. The terms $\gamma^{-}$and $\gamma^{+}$denote then the left and the right limit of the velocity function $t \rightarrow \gamma(t)$ which has a discontinuity at the time of collision $t^{\star}$. Internal oscillations are not possible in rigid bodies. A choice $\varepsilon<1$ of the restitution coefficient therefore implies the assumption of an instantaneous thermal loss of energy for the rigid body model.

Regarding the one-dimensional problem of two colliding particles, the Newtonian impact law (11) can be derived from conservation of linear momentum and additional energetic assumptions. The law itself has not to be regarded as an independent principle, but as an equation to specify the amount of dissipation of the system with respect to the chosen spatial discretization. When using Newton's impact law (11), one always has to carefully check the rigid body assumption. Special attention has to be paid if the rigid model is a significant simplification of a complex highly dimensional dynamic system, for which an energy transfer to inner degrees of freedom might influence drastically the collision behavior. Examples are effects of wave propagation, which will be addressed in the next section. 


\section{Example: Collision of two elastic rods}

In this section we discuss in detail the collision behavior of two homogenous elastic rods (Young's modulus $E$, specific mass $\rho$, cross section $A$, length $l_{1}<l_{2}$ ). Rod 1 moves with constant velocity $v$ towards rod 2 which is at rest. On the basis of this example taken from [2], it will be shown that the restitution coefficient depends on the spatial discretization depth of the individual bodies and must therefore not be regarded as a material pairing constant only.

As a first step, the example is treated in the framework of the theory of linear elasticity. The bodies are modeled as continua with distributed degrees of freedom. This corresponds to an "infinitely" fine discretization of the system consisting of the two rods. The problem is solved by the wave equation. Let $u(x, t)$ be the longitudinal displacement of the rod's physical point $x$ at time $t, \varepsilon(x, t)=u_{x}(x, t)$ the local strain in the rods, and $\sigma(x, t)$ the associated stress that result from the constitutive law $\sigma(x, t)=E \varepsilon(x, t)$. Let further $x \in\left[l_{1}+l_{2}\right]$ to address the physical points of rod 1 by $x \in\left[0, l_{1}\right)$ and those of rod 2 by $x \in\left(l_{1}, l_{1}+l_{2}\right]$, see Fig. 2. By setting $t:=0$ as the time at which the rods get into contact, we may restrict the time interval of interest for the collision to $t \geq 0$. The dynamic behavior of the rods is described by the wave equation

$u_{t t}(x, t)=c^{2} u_{x x}(x, t), \quad c^{2}=\frac{E}{\rho}$,

where $c$ is the wave propagation velocity.

In order to obtain a well-defined problem, we first set up completely the pre-collision state of the two rods. We assume both rods to get in contact at time $t=0$ and assign the associated displacements at the contact the value zero,

$u\left(l_{1}^{-}, 0\right)=u\left(l_{1}^{+}, 0\right)=0$.

The stress distribution of both rods are assumed equal to zero prior to collision,

$u_{x}(x, 0)=0 \quad \forall x \in\left[0, l_{1}\right) \cup\left(l_{1}, l_{1}+l_{2}\right]$.

Before the collision, rod 1 moves with constant velocity $v$ towards rod 2 which itself is at rest. This results in the velocity field

$\begin{array}{ll}u_{t}(x, 0)=v & \forall x \in\left[0, l_{1}\right), \\ u_{t}(x, 0)=0 & \forall x \in\left(l_{1}, l_{1}+l_{2}\right]\end{array}$

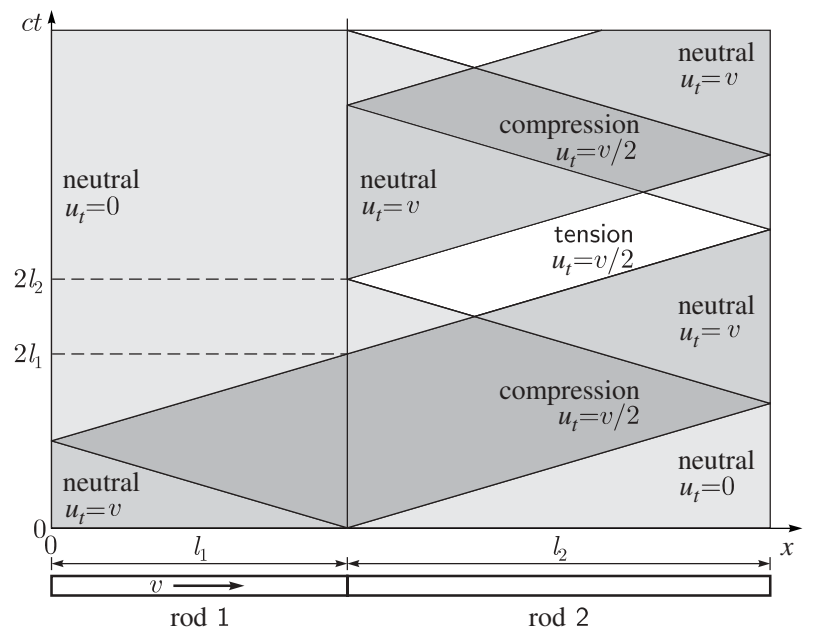

Fig. 2. Wave propagation in the rods during the collision process 
at the start of the collision. It is worth mentioning that the point $x=l_{1}$ has been excluded in Eqs. (13)-(15), because discontinuities have to be expected there.

The displacement boundary conditions at the investigated time interval $t>0$ are points free of stresses at $x=0$ and $x=l_{1}+l_{2}$,

$u_{x}(0, t)=u_{x}\left(l_{1}+l_{2}, t\right)=0 \quad \forall t>0$.

There is also a unilateral constraint $u\left(l_{1}^{+}, t\right)-u\left(l_{1}^{-}, t\right) \geq 0$ at $x=l_{1}$ which describes on displacement level the state of the contact. One has to distinguish between two cases if this unilateral constraint is stated on velocity level: If the contact is open, we have again endpoints which are free of stresses,

$u\left(l_{1}^{+}, t\right)-u\left(l_{1}^{-}, t\right)>0 \quad \Rightarrow \quad u_{x}\left(l_{1}^{+}, t\right)=u_{x}\left(l_{1}^{-}, t\right)=0 \quad \forall t>0$.

If the contact is closed, the state of the constraint is described by a complementarity condition. It expresses the only compressive character of the stresses for the closed contact, and the property of "signed" velocities at the moment of separation,

$$
\begin{aligned}
u\left(l_{1}^{+}, t\right)-u\left(l_{1}^{-}, t\right)=0: & u_{x}\left(l_{1}^{+}, t\right)=u_{x}\left(l_{1}^{-}, t\right) \leq 0, \\
& u_{t}\left(l_{1}^{+}, t\right)-u_{t}\left(l_{1}^{-}, t\right) \geq 0, \\
& u_{x}\left(l_{1}^{ \pm}, t\right)\left(u_{t}\left(l_{1}^{+}, t\right)-u_{t}\left(l_{1}^{-}, t\right)\right)=0 \quad \forall t>0 .
\end{aligned}
$$

As a final and independent condition, not being contained in any of the above expressions and not being given in any axiomatic way, an impact law has heuristically to be introduced, which describes the velocity jump at the moment of collision at the contact. We choose

$u_{t}\left(l_{1}^{+}, 0^{+}\right)=u_{t}\left(l_{1}^{-}, 0^{+}\right)$,

which corresponds by (11) with $\gamma(t)=u_{t}\left(l_{1}^{+}, t\right)-u_{t}\left(l_{1}^{-}, t\right)$ to a completely inelastic impact model, for which the relative velocity of the two bodies disappears after the impact.

Equations (12)-(19) completely describe the impact problem and can easily be solved with the characteristics of the system at hand. The resulting temporal and spatial behavior of the two rods is depicted in Fig. 2. The regions marked as tension, compression and neutral represent in the same order that $u_{x}=v / 2 c, u_{x}=-v / 2 c$ and $u_{x}=0$, respectively. One notices that the point of $\operatorname{contact} x=l_{1}$ is under compression in the interval $0<c t<2 l_{1}$. This implies that (18) holds in the form $u_{x}\left(l_{1}^{ \pm}, t\right)<0$ and $u_{t}\left(l_{1}^{+}, t\right)=u_{t}\left(l_{1}^{-}, t\right)$. At time $c t=2 l_{1}$, the equalization process between the rods, during which waves pass across the contact, is completed. The left rod remains in an state free of stress, whereas in the right rod a wave pattern evolves with period $c t$ and wavelength $2 l_{2}$. In the interval $2 l_{1}<c t<2 l_{2}$ the contact is still closed but already free of stress. Equation (18) takes here the form $u_{x}\left(l_{1}^{ \pm}, t\right)=0$ and $u_{t}\left(l_{1}^{+}, t\right)=u_{t}\left(l_{1}^{-}, t\right)$. At time $c t=2 l_{2}$ a tensile wave reaches the point of contact, but cannot pass it because of the inequality $u_{x}\left(l_{1}^{ \pm}, t\right) \leq 0$. As a consequence, the contact opens with $u_{x}\left(l_{1}^{ \pm}, t\right)=0$ and $u_{t}\left(l_{1}^{+}, t\right)-u_{t}\left(l_{1}^{-}, t\right)=v>0$. For $c t>2 l_{2}$ Eq. (17) is valid, which describes the open contact.

An energetic analysis shows that the system is non-dissipative, i.e., that the sum of kinetic energy $T(t)$ and potential energy $V(t)$ is constant during the entire collision process. Note in particular that we have used by (19) the model of a completely inelastic impact for the collision. However, this collision turns out to be non-dissipative, based on the fact that the measure of the local masses which are to be instantaneously decelerated during impact is zero. We have just before the collision $T_{1}\left(0^{-}\right)=1 / 2 \rho A l_{1} v^{2}, T_{2}\left(0^{-}\right)=0, V_{1}\left(0^{-}\right)=V_{2}\left(0^{-}\right)=0$. After the completion of the wave equalization process $c t>2 l_{1}$, we have $T_{1}(t)=V_{1}(t)=0$ and $T_{2}(t)+V_{2}(t)=T_{1}\left(0^{-}\right)$, where $T_{2}(t)$ and $V_{2}(t)$ vary $2 l_{1}$-periodically in $c t$. 
As a second step we model the system as two discrete masses $m_{1}=\rho A l_{1}, m_{2}=\rho A l_{2}$ and treat the impact by the method introduced in Sect. 2. The restitution coefficient $\varepsilon$ in (11) shall be determined such that the results obtained by considering the system as a continuum as in Fig. 2 agree with the ones from the discretized version on average. This means that the velocities of the centers of mass $v_{1}^{+}, v_{2}^{+}$of both rods are obtained correctly in the sense of the wave equation (12) from (11) and (3). The velocities of the centers of mass of both rods just before the impact are $v_{1}^{-}=v$ and $v_{2}^{-}=0$. After the impact one obtains from Fig. 2 for rod 1 directly $v_{1}^{+}=0$. The velocity $v_{2}^{+}$results in $v_{2}^{+}=l_{1} / l_{2} v$ and can be obtained either by evaluating the conservation of linear momentum $p^{+}=p^{-}$with $p$ according to (1), or from Fig. 2 by spatial averaging over $\left[l_{1}, l_{1}+l_{2}\right] \ni x$ for any fixed time $c t \geq 2 l_{1}$. The relative velocities are then

$\gamma^{-}=v_{2}^{-}-v_{1}^{-}=-v, \quad \gamma^{+}=v_{2}^{+}-v_{1}^{+}=\frac{l_{1}}{l_{2}} v$,

which leads to a restitution coefficient

$\varepsilon=\frac{l_{1}}{l_{2}}$

when (11) is evaluated. This examples illustrates that the restitution coefficient cannot only be considered as a material pairing constant. As we have seen, it depends on the ratio of the lengths or, in other words, on the geometry of the colliding bodies [2]. It includes in an extracted manner the total wave propagation process that occurs during the impact. The waves that continue to propagate in the right rod after the collision has been completed, are beyond our chosen spatial resolution and must therefore be understood as dissipation. With (8) and (20), (21) one obtains here

$T^{+}-T^{-}=\frac{1}{2} \frac{l_{1}}{l_{2}} \rho A v^{2}\left(l_{1}-l_{2}\right)<0$.

The term "dissipation" has therefore to be used and understood in the context of the spatial discretization depth of the mechanical system and includes also energy transfer to degrees of freedom that have not been incorporated in the model. The duration $c t=2 l_{2}$ of the collision obtained by the continuum model provides, by the way, a reasonable lower limit for the length of a time interval to be used to locate the impact numerically in a rigid body approach: Any attempt to determine a more exact impact time does not make sense from the physics point of view.

\section{Systems with a single collision point}

In Sect. 2, the impact law has been obtained from the limiting cases of energy conservation and maximum dissipation at the collision. It shall now be assessed to what extend these both concepts can be used to treat single-point impacts in a multi-body system. We consider systems as depicted in Fig. 3, consisting of rigid bodies that are linked together by perfect bilateral constraints. We assume that only two out of these bodies may collide with each other, contacting each other in a single point only.

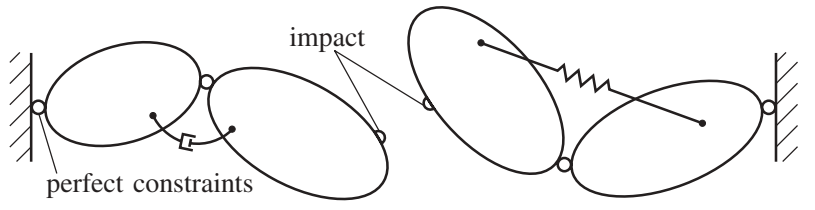

Fig. 3. Multi-body system with a single point of collision 
For classical impact-free systems, one has to use for each body separately Newton's second law and Euler's axiom to derive the equations of motion. The conservation principles of linear and angular momentum will not suffice, because the rigid bodies in the system interact with each other by external forces. This procedure has to be applied to impact problems in the same fashion, because external impulsive forces are as natural as classical non-impulsive forces for impact-free motion. In order to accomplish this, the equations of motion have to be replaced by their integrated form, which we call the impact equations [3], [4]. Integration has to be performed over a single element, the impact time. Only atomic terms, i.e., measures of Dirac type contribute, such as impulsive forces and drastic changes in the accelerations which lead to velocity jumps. For every external force, whether conventional or impulsive, a force direction has to be specified in the model in accordance with the physical behavior of the system. In addition, a force law has to be established in order to calculate the values of the forces from the kinematic state of the system. In our case of the single-point collision, this requires a collision model [3], [4] which is depicted in Fig. 4.

The left part of Fig. 4 describes the kinematics of the collision. The contact points $P$ and $Q$ at which the collision takes place are defined as the surface points that lie on a connecting line perpendicular to both of the body contours. For $\mathbf{n}$ being the unit normal vector pointing inwards at point $P$ of the left body, the distance $g$ of the collision points becomes

$g=\mathbf{n}^{T}\left(\mathbf{r}_{P}-\mathbf{r}_{Q}\right) \geq 0$.

For $g>0$ both bodies are spatially separated from each other. The case $g=0$ describes the situation, where both bodies touch each other at the points $P$ and $Q$, as at the time instant of the collision. If (23) is differentiated with respect to time, then one obtains $\dot{g}=\gamma$ as the velocity

$\gamma=\mathbf{n}^{T}\left(\mathbf{v}_{P}-\mathbf{v}_{Q}\right)$,

with which the points of collision move relative to each other in $n$-direction. In (24), $\mathbf{v}_{P}$ and $\mathbf{v}_{Q}$ are the velocities of the rigid body contour points, which momentarily coincide with $P$ and $Q$. The force interaction model is depicted on the right side of Fig. 4. It is assumed that the impulsive forces at the impact act at points $P$ and $Q$ in the direction of $\mathbf{n}$, which corresponds to a frictionless contact behavior. Due to the law of interaction one has $\mathscr{F}_{P}=-\mathscr{F}_{Q}$, and therefore

$\mathscr{F}_{P}=\mathbf{n} \Lambda, \quad \mathscr{F}_{Q}=-\mathbf{n} \Lambda \quad(\Lambda \geq 0)$.

The scalar $\Lambda$ is the value of the force impulse that occurs during collision. It is nonnegative if, on average, positive (compressive) forces act during the time interval of the real physical collision.

In order to fulfill the impact equations for all $n$ bodies in the system, i.e., the equations obtained by integration of Newton's second law and Euler's axiom over the impact time, we demand that the corresponding virtual work expression is zero for all virtual linear and angular velocities at the impact. Similar to the case of motion without impact, the virtual work has to be composed of all instantaneous changes in the linear and angular momenta as well as all external forces and moments that might have impulsive character. Bounded forces or gyroscopic acceleration
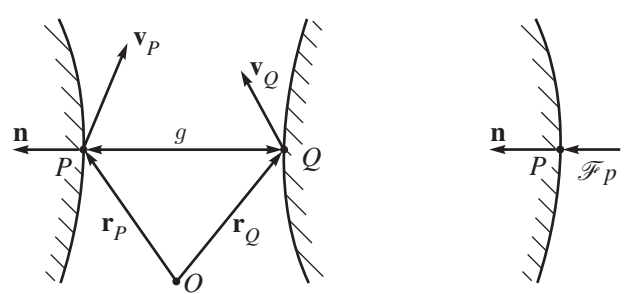

Fig. 4. Kinematics and forces during the collision impulsive 
components do not need to be considered, since they do not contribute to the impacts equations after integrating over a singleton in time and drop out of the equations as a consequence. The dynamic equilibrium at the impact is thus expressed by the variational equation

$$
\begin{aligned}
\sum_{i=1}^{n} & {\left[\delta \mathbf{v}_{S}^{T}\left(m\left(\mathbf{v}_{S}^{+}-\mathbf{v}_{S}^{-}\right)-\mathscr{F}_{S}\right)+\delta \Omega^{T}\left(\Theta_{S}\left(\Omega^{+}-\Omega^{-}\right)-\mathscr{M}_{S}\right)\right]_{i} } \\
& -\delta \mathbf{v}_{P}^{T} \mathscr{F}_{P}-\delta \mathbf{v}_{Q}^{T} \mathscr{F}_{Q}=0 \quad \forall \delta \mathbf{v}_{\star}, \delta \Omega .
\end{aligned}
$$

Here, $m_{i}$ and $\Theta_{S i}$ are the masses and the symmetric positive definite inertia operators of the $n$ rigid bodies in the system, $\mathbf{v}_{S i}$ and $\Omega_{i}$ are the velocities of the centers of mass and the angular velocities of the bodies, and $\mathscr{F}_{S i}$ and $\mathscr{M}_{i}$ are all the external forces and moments of probable impulsive nature. With exception of the impulsive forces, whose virtual work is considered in the second line of (26), these are solely the constraint forces of the bilateral constraints in the system. The upper indices + and - identify, as in Sect. 2, the state directly before and after the impact. The lower index $\star$ in the virtual velocities generally represents any of the points $P, Q$ and $S$.

For further discussions we formulate now the collision problem in the configuration space of the mechanical system. Let $\mathbf{q} \in \mathbb{R}^{f}$ be local coordinates of the $f$-dimensional configuration manifold set up through the bilateral constraints, and let $\mathbf{u}=\dot{\mathbf{q}}$ be the associated velocities. The coordinates $\mathbf{q}$ are classical minimal coordinates for the case that the collision contact is open $(g>0)$. For scleronomic systems, both, the real and the virtual velocities (26) transform according to

$$
\begin{array}{ll}
\mathbf{v}_{\star}=\mathbf{J}_{\star}(\mathbf{q}) \mathbf{u}, & \delta \mathbf{v}_{\star}=\mathbf{J}_{\star}(\mathbf{q}) \delta \mathbf{u}, \\
\Omega=\mathbf{J}_{R}(\mathbf{q}) \mathbf{u}, & \delta \Omega=\mathbf{J}_{R}(\mathbf{q}) \delta \mathbf{u},
\end{array}
$$

where $\mathbf{J}_{R}(\mathbf{q})$ and $\mathbf{J}_{\star}(\mathbf{q})$ are the Jacobians of rotation and of translation in the points $\star \in\{P, Q, S\}$. By the impenetrability condition (23) a subset of $\mathbb{R}^{f}$ is obtained which is nonconvex in general,

$\mathscr{C}:=\left\{\mathbf{q} \mid g(\mathbf{q})=\mathbf{n}^{T}\left(\mathbf{r}_{P}-\mathbf{r}_{Q}\right) \geq 0\right\}$.

This subset defines the kinematically admissible coordinates $\mathbf{q}$ and further restricts the system to a configuration manifold with boundary. If the collision points are placed in addition such that the common tangent plane of both bodies is uniquely determined in the case of contact, then we may assume the boundary in the neighborhood of the impact coordinates $\mathbf{q} \in \partial \mathscr{C}$ as smooth. The relative velocity $\gamma$ normal to the surface of the impacting bodies (24) becomes with (27)

$\gamma=\mathbf{n}^{T}\left(\mathbf{J}_{P}-\mathbf{J}_{Q}\right) \mathbf{u}$.

On the other hand, $\gamma(t)=\dot{g}(\mathbf{q}(t))=(\partial g / \partial \mathbf{q}) \mathbf{u}(t)$, enables us to identify, by comparing this expression with (29), the coordinates of the differential of $g$ as

$\frac{\partial g}{\partial \mathbf{q}}=\mathbf{n}^{T}\left(\mathbf{J}_{P}-\mathbf{J}_{Q}\right)$

Considering (27) the virtual work expression (26) becomes

$$
\begin{aligned}
& \delta \mathbf{u}^{T} \sum_{i=1}^{n}\left(\mathbf{J}_{S}^{T} m \mathbf{J}_{S}+\mathbf{J}_{R}^{T} \Theta_{S} \mathbf{J}_{R}\right)_{i}\left(\mathbf{u}^{+}-\mathbf{u}^{-}\right)-\delta \mathbf{u}^{T} \sum_{i=1}^{n}\left(\mathbf{J}_{S}^{T} \mathscr{F}_{S}+\mathbf{J}_{R}^{T} \mathscr{M}_{S}\right)_{i} \\
& \quad-\delta \mathbf{u}^{T}\left(\mathbf{J}_{P}^{T}-\mathbf{J}_{Q}^{T}\right) \mathbf{n} \Lambda=0 \quad \forall \delta \mathbf{u} .
\end{aligned}
$$


We extend now the classical definition of perfect bilateral constraints to impacts by demanding the virtual work of the impulsive constraint forces to vanish for any geometrically compatible virtual velocities $\delta \mathbf{u}$,

$\delta \mathbf{u}^{T} \sum_{i=1}^{n}\left(\mathbf{J}_{S}^{T} \mathscr{F}_{S}+\mathbf{J}_{R}^{T} \mathscr{M}_{S}\right)_{i}=0 \quad \forall \delta \mathbf{u}$.

By this definition, we may now cross out the impulsive constraint forces (32) from (31). This concept has not to be taken as an axiom, but merely expresses our wish to keep the classical orthogonality property between the constraint forces and the surfaces of constrained motion also for impulsive behavior. Perfect bilateral constraints are basically used in the modeling of joints and guidances if the influence of Coulomb friction is negligible. According to Eq. (32), this property has now to be checked at the stage of modeling also for the impulsive constraint forces.

With Eqs. (28)-(32) all relations are now available to state the collision problem on the configuration manifold of the system. These are, in condensed form, Eq. (28) which defines the set of admissible displacements

$\mathscr{C}=\{\mathbf{q} \mid g(\mathbf{q}) \geq 0\}$,

the virtual work expression (31) with the external impulsive forces originating from the bilateral constraints (32), and the normal relative velocity (29). By taking into account (30) and by setting $\mathbf{M}(\mathbf{q}):=\sum_{i=1}^{n}\left(\mathbf{J}_{S}^{T} m \mathbf{J}_{S}+\mathbf{J}_{R}^{T} \Theta_{S} \mathbf{J}_{R}\right)_{i}$ for the mass matrix of the system, we obtain (31) and (29) in the form:

$\mathbf{M}\left(\mathbf{u}^{+}-\mathbf{u}^{-}\right)=\left(\frac{\partial g}{\partial \mathbf{q}}\right)^{T} \Lambda, \quad \gamma=\frac{\partial g}{\partial \mathbf{q}} \mathbf{u}$.

The left equation in (34) is called the impact equation and should not be confused with the collision law, which has not yet been introduced.

For a geometric interpretation of (34) we map all the vectors defined in the cotangent space to the tangent space by the use of the natural isomorphism induced by the kinetic metric. The inner product of the associated coordinates in the tangent space is denoted by a dot, $\mathbf{a} \cdot \mathbf{b}:=\mathbf{a}^{T} \mathbf{M b}$, and the associated norm is written as $\|\mathbf{a}\|:=\sqrt{\mathbf{a} \cdot \mathbf{a}}$. With

$\nabla g=\mathbf{M}^{-1}\left(\frac{\partial g}{\partial \mathbf{q}}\right)^{T}$

the coordinates of the gradient of $g(\mathbf{q})$ in the tangent space, we get

$\mathbf{u}^{+}-\mathbf{u}^{-}=\nabla g \Lambda, \quad \gamma=\nabla g \cdot \mathbf{u}$.

With arbitrary $\Lambda \geq 0$ (25), the right-hand side of the impact equation describes a half-line $\mathscr{T}_{\mathscr{L}}^{\perp}(\mathbf{q})$ in the tangent space at the impact point $\mathbf{q} \in \partial \mathscr{C}$. We may now define an object $\mathscr{T}_{\mathscr{C}}(\mathbf{q})$ via

$\mathscr{T}_{\mathscr{C}}^{\perp}(\mathbf{q}):=\{-\nabla g(\mathbf{q}) \Lambda \mid \Lambda \geq 0\}, \quad \mathscr{T}_{\mathscr{C}}(\mathbf{q}):=\left\{\mathbf{z} \mid \mathbf{z} \cdot \mathbf{z}^{\perp} \leq 0 \quad \forall \mathbf{z}^{\perp} \in \mathscr{T}_{\mathscr{C}}^{\perp}(\mathbf{q})\right\}$,

which is a half-space orthogonal to the half-line $\mathscr{T}_{\mathscr{C}}(\mathbf{q})$, see Fig. 5. Its boundary $\partial \mathscr{T}_{\mathscr{C}}(\mathbf{q})$ is the tangent space associated with the sub-manifold $\{\mathbf{q} \mid g(\mathbf{q})=0\}$ in point $\mathbf{q}$. This half-space expresses the kinematic compatibility which the post-impact velocity $\mathbf{u}^{+}$has to comply with, in order to keep the system's trajectory $\mathbf{q}(t)$ within the set of admissible displacements $\mathscr{C}$ and to prevent interpenetration of the colliding bodies. So far, $\mathbf{u}^{+}$has to fulfill two requirements: the already discussed kinematic compatibility $\mathbf{u}^{+} \in \mathscr{T}_{\mathscr{C}}(\mathbf{q})$ and the impact equation in (36), $\mathbf{u}^{+} \in \mathbf{u}^{-}-\mathscr{T}_{\mathscr{C}}^{\perp}(\mathbf{q})$. 


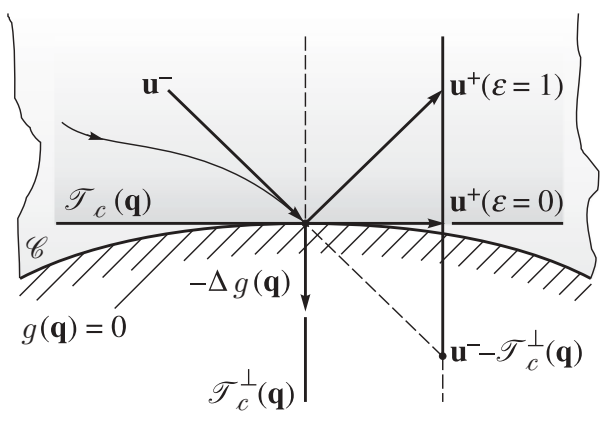

Fig. 5. Geometry of the single contact collision without friction

Consider now a trajectory which reaches the boundary of the configuration manifold under a velocity $\mathbf{u}^{-} \in-\mathscr{T}_{\mathscr{C}}(\mathbf{q})$. It is then possible to derive limits on the velocity $\mathbf{u}^{+}$after the impact by using the same concepts as in Sect. 2, i.e., the concepts of energy conservation and maximal dissipation. With the kinetic energy $T=\frac{1}{2}\|\mathbf{u}\|^{2}$ for a scleronomic system and the impact equations in (36), the following relation for non-dissipative impacts can be derived:

$T^{+}=T^{-} \quad \Rightarrow \quad\left\|\mathbf{u}^{+}\right\|=\left\|\mathbf{u}^{-}\right\| \quad \Rightarrow \quad \mathbf{u}^{+}=-\mathbf{u}^{-}$, if $\mathbf{u}^{+} \in \mathbf{u}^{-}-\mathscr{T}_{\mathscr{L}}^{\perp}(\mathbf{q})$.

The case $\mathbf{u}^{+}=\mathbf{u}^{-}$has to be excluded because kinematic compatibility would be violated. The impact behavior (38) represents a reflection of the velocity $\mathbf{u}^{-}$on the hyperplane defined by the boundary of $\mathscr{T}_{\mathscr{C}}(\mathbf{q})$. Due to (38), the relative velocity $\gamma$ from (36) is again inverted,

$\gamma^{+}=\nabla g \cdot \mathbf{u}^{+}=-\nabla g \cdot \mathbf{u}^{-}=-\gamma^{-}$.

On the other hand, one can uniquely reconstruct $\mathbf{u}^{+}$from $\mathbf{u}^{-}$by using (36) when $\gamma^{+}=-\gamma^{-}$is given.

The principle of maximum dissipation incorporates the minimization of the post-impact kinetic energy under consideration of the laws of impact and provides $\mathbf{u}^{+}$as the nearest point on $\mathbf{u}^{-}-\mathscr{T}_{\mathscr{C}}^{\perp}(\mathbf{q})$ to the origin, as it can be easily seen in Fig. 5 . This corresponds to an orthogonal projection of $\mathbf{u}^{-}$on the boundary of $\mathscr{T}_{\mathscr{C}}(\mathbf{q})$. Kinematic consistency is then automatically guaranteed. The principle of maximal dissipation may thus be stated as follows:

Minimize $\left\|\mathbf{u}^{+}\right\|$under $\mathbf{u}^{+} \in \mathbf{u}^{-}-\mathscr{T}_{\mathscr{C}}^{\perp}(\mathbf{q}) \quad \Rightarrow \quad \mathbf{u}^{+}=\operatorname{proj}_{\partial \mathscr{T}_{\mathscr{G}}(\mathbf{q})}\left(\mathbf{u}^{-}\right)$.

For the relative velocity after the impact we obtain

$\gamma^{+}=\nabla g \cdot \mathbf{u}^{+}=0$,

due to the orthogonality of $\mathbf{u}^{+}$and $\nabla g$. Again, the post-impact velocity $\mathbf{u}^{+}$is uniquely determined by $\mathbf{u}^{-}$and (36) for known $\gamma^{+}=0$.

By introducing now a restitution coefficient $\varepsilon$ as in Sect. 2, i.e.,

$\gamma^{+}=-\varepsilon \gamma^{-} \quad(0 \leq \varepsilon \leq 1)$,

one is able to access also dissipative behavior of the collision which lies between the two limit cases mentioned. For a single-contact collision, Newton's impact law can again be deduced from energetic considerations, if the structure outlined in Eq. (34) is provided for the mechanical system. Besides the definition of the constraint forces (32) which has been extended to impacts, the substantial physical assumption leading to Eqs. (34) consists in the equality of the differential $\partial g / \partial \mathbf{q}$ occurring in the relative velocity $\gamma=\partial g / \partial \mathbf{q}$ with the generalized direction $\mathbf{w}$ of the impulsive force in the impact equations $\mathbf{M}\left(\mathbf{u}^{+}-\mathbf{u}^{-}\right)=\mathbf{w} \Lambda$, i.e., $\mathbf{w}^{T}=\partial g / \partial \mathbf{q}$. This property is used in [5] as the definition of a unilateral perfect holonomic constraint and plays a role in non-smooth analytical mechanics as important as the corresponding concept in the classical case. The essence of the single-contact collision lies, again, not in the impact law 


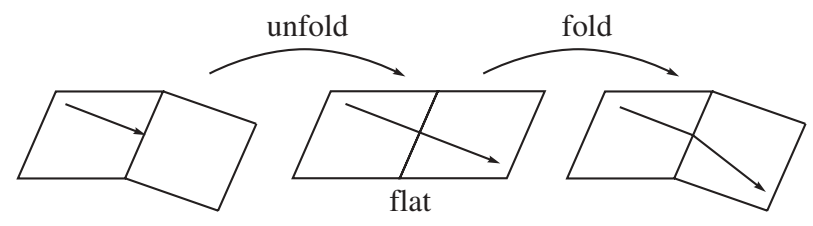

Fig. 6. Natural extension of a geodesic at a sharp edge

but in the generalized concept of perfect constraints extended to impacts and inequality constraints. Note finally that conservation of the generalized impulses applies in the $f-1$ directions orthogonal to $\nabla g(\mathbf{q}), \mathbf{M} \operatorname{proj}_{\partial \mathscr{T}_{8}(\mathbf{q})}\left(\mathbf{u}^{+}\right)=\mathbf{M} \operatorname{proj}_{\partial \mathscr{T}_{\mathscr{\zeta}}(\mathbf{q})}\left(\mathbf{u}^{-}\right)$, which generalizes all results from Sect. 2.

An impact problem related to the single-contact collision is the natural extension of a geodesic on a manifold with a sharp edge, which, as in Fig. 6, can uniquely be obtained by a local straight and back folding. In order not to leave the manifold at the sharp edge, the (nonvanishing) velocity of the trajectory has again instantaneously be changed when the edge is transversally approached. The result shown in Fig. 6 can also be achieved by using a reflecting hyperplane, which is aligned with the angle bisector and therefore is unique. This procedure connects sharp edges to the non-dissipative collisions in Fig. 5, for which the manifold is bend over by 180 degrees. For certain mechanical systems, the crossing of a sharp bend can actually be interpreted as a non-dissipative collision, as the example of a coach driven on a plain track from [6]. It is important to notice that one can continue working in a natural way without the introduction of additional impact laws, even in the presence of sharp bends.

\section{Multi-contact systems}

We consider now multi-contact rigid body systems, or more generally, multi-contact systems with finite degrees of freedom, for example generated by discretization of continua. Typical examples are the rocking rod with two contacts, see Fig. 7, or the Newton's cradle with four contacts. For the mechanical system, the same assumptions are taken as in Sect. 4, especially those of the perfect bilateral constraints (32) and those of the collision model in Fig. 4. Unilateral perfect constraints are defined in the same fashion, but now extended to $m$ contacts.

To formulate the impact problem, Eqs. (33)-(37) from Sect. 4 can be transferred directly to multi-contacts. The set $\mathscr{C}$ of admissible displacements for $m$ unilateral constraints $g_{i}$ of the form (23) arises now in analogy to (33) as intersection,

$\mathscr{C}=\left\{\mathbf{q} \mid g_{i}(\mathbf{q}) \geq 0, \quad i=1, \ldots, m\right\}$.

Further let $\mathscr{H}$ be the index set of the closed contacts,

$\mathscr{H}:=\left\{i \mid g_{i}(\mathbf{q})=0\right\}$,

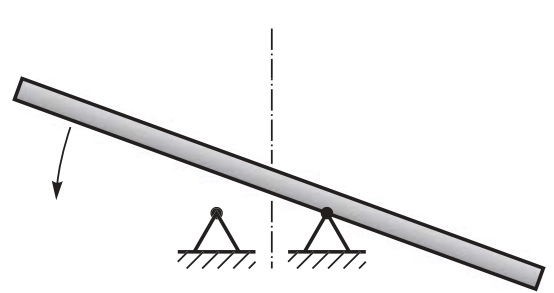

rocking rod

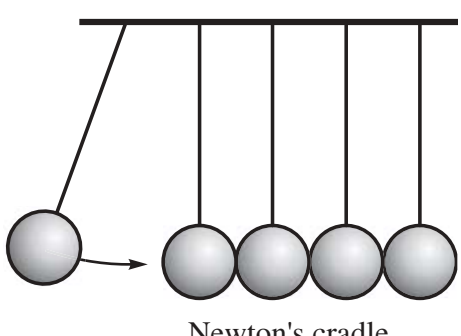

Newton's cradle

Fig. 7. Examples of multi-contact collision problems 
because only those can take part in the collision and therefore can carry impulses. The normal relative velocities $\gamma_{i}$ are defined as in (29) and can be displayed as in (34) or (36),

$\gamma_{i}=\frac{\partial g_{i}}{\partial \mathbf{q}} \mathbf{u}=\nabla g_{i} \cdot \mathbf{u}$.

The impulses of all closed contacts are now to be considered in the impact equations (34) and (36), thus

$\mathbf{M}\left(\mathbf{u}^{+}-\mathbf{u}^{-}\right)=\sum_{i \in \mathscr{H}}\left(\frac{\partial g_{i}}{\partial \mathbf{q}}\right)^{T} \Lambda_{i} \quad$ resp. $\quad \mathbf{u}^{+}-\mathbf{u}^{-}=\sum_{i \in \mathscr{H}} \nabla g_{i} \Lambda_{i} \quad\left(\Lambda_{i} \geq 0\right)$.

This does not yet mean, that all those contacts really take impulses. With $\Lambda_{i} \geq 0$, the total impulsive force at the impact displayed in the tangent space, i.e., the right-hand side of the second equation in (46), is composed as a nonnegative linear combination of the gradients $\nabla g_{i}$. The set of all such possible linear combinations generates a convex cone and is denoted by $-\mathscr{T}_{\mathscr{C}}^{\perp}(\mathbf{q})$ in analogy to the half-line in (37),

$\mathscr{T}_{\mathscr{C}}^{\perp}(\mathbf{q}):=\left\{\mathbf{z}^{\perp} \mid \mathbf{z}^{\perp}=-\sum_{i \in \mathscr{H}} \nabla g_{i}(\mathbf{q}) \Lambda_{i}, \Lambda_{i} \geq 0\right\}$.

The cone $\mathscr{T}_{\mathscr{C}}(\mathbf{q})$ orthogonal to (47), which approximates the configuration manifold in a neighborhood of the impact point and which represents the kinematic compatible velocities, is defined as in the right equation in (37) by

$\mathscr{T}_{\mathscr{C}}(\mathbf{q}):=\left\{\mathbf{z} \mid \mathbf{z} \cdot \mathbf{z}^{\perp} \leq 0 \quad \forall \mathbf{z}^{\perp} \in \mathscr{T}_{\mathscr{C}}^{\perp}(\mathbf{q})\right\}$.

As a last condition, the energetic consistency at impact has to be guaranteed. For scleronomic systems, the kinetic energy is $T=1 / 2\|\mathbf{u}\|^{2}$. Energy gain at impact can be excluded by claiming that possible velocities $\mathbf{u}^{+}$are not allowed to lie outside a ball $\mathscr{B}_{\mathbf{u}^{-}}(\mathbf{q})$ with radius $\left\|\mathbf{u}^{-}\right\|$. Thus the set of energetically consistent velocities after impact is

$\mathscr{B}_{\mathbf{u}^{-}}(\mathbf{q}):=\left\{\mathbf{y}\|\mathbf{y}\| \leq\left\|\mathbf{u}^{-}\right\|\right\}$.

The subsets of the tangent space at the collision point defined in (47)-(49) correspond to three necessary conditions for the velocities after impact $\mathbf{u}^{+}$. These are in turn the impact equation, the kinematic compatibility and the energetic consistency,

$\mathbf{u}^{+} \in \mathbf{u}^{-}-\mathscr{T}_{\mathscr{C}}^{\frac{1}{\mathscr{C}}}(\mathbf{q}), \quad \mathbf{u}^{+} \in \mathscr{T}_{\mathscr{C}}(\mathbf{q}), \quad \mathbf{u}^{+} \in \mathscr{B}_{\mathbf{u}^{-}}(\mathbf{q})$.

As intersection $\mathscr{S}_{\mathbf{u}^{-}}(\mathbf{q})$ we obtain a convex subset of the energy ball, in case of a collision problem with two active contacts drawn dark-gray in Fig. 8. In contrast to a single-contact

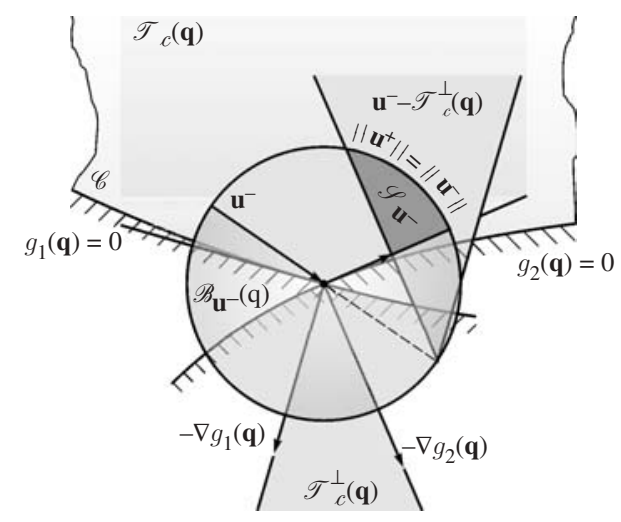

Fig. 8. Geometry of the multi-contact collision without friction 
impact (Fig. 5), the post-impact velocities are no longer restricted to a line segment only, but are taken from a considerably bigger set of higher dimension.

As in Sects. 2 and 4, we want to analyze the two special cases "impact without loss" and "maximal dissipation". For impacts without loss, the boundary of the energy ball, the energy sphere, is to be taken for the intersection of the sets addressed in (50), thus

$\mathbf{u}^{+} \in \mathbf{u}^{-}-\mathscr{T}_{\mathscr{C}}^{\perp}(\mathbf{q}) \cap \mathscr{T}_{\mathscr{C}}(\mathbf{q}) \cap \partial \mathscr{B}_{\mathbf{u}^{-}}(\mathbf{q})$.

We recognize immediately from Fig. 8 that this does not lead to a uniquely determined velocity $\mathbf{u}^{+}$any more, but to a region on the energy sphere bounded by the (translated) cones $-\mathscr{T} \frac{1}{\mathscr{C}}(\mathbf{q})$ and $\mathscr{T}_{\mathscr{C}}(\mathbf{q})$. All elements of this subset of $\partial \mathscr{B}_{\mathbf{u}^{-}}(\mathbf{q})$ are thus equal candidates for $\mathbf{u}^{+}$.

In order to analyze the collision event under maximum dissipation, we formulate it as a minimization problem on the difference in kinetic energies and consider as constraints the impact equation, i.e., the first equation in (50). One obtains the quadratic program:

Minimize $f\left(\mathbf{u}^{+}\right):=T^{+}-T^{-}=\frac{1}{2}\left\|\mathbf{u}^{+}\right\|^{2}-\frac{1}{2}\left\|\mathbf{u}^{-}\right\|^{2}$ under $\mathbf{u}^{+} \in \mathbf{u}^{-}-\mathscr{T}_{\mathscr{C}}^{\perp}(\mathbf{q})$,

which has a unique solution $\mathbf{u}^{+}$, because it deals with a strictly convex function $f\left(\mathbf{u}^{+}\right)$to be minimized on a convex set $\mathbf{u}^{-}-\mathscr{T}_{\mathscr{C}}^{\perp}(\mathbf{q})$ [7]. In [8]-[10] it is shown that this solution $\mathbf{u}^{+}$is the closest point in the set $\mathscr{T}_{\mathscr{C}}(\mathbf{q})$ to $\mathbf{u}^{-}$, see Fig. 8, which is normally called a proximation [7] and denoted by

$\mathbf{u}^{+}=\operatorname{prox}_{\mathscr{T}_{\mathscr{G}}(\mathbf{q})}\left(\mathbf{u}^{-}\right)$.

Energetic consistency is always guaranteed for this solution [8], which is obvious, regarding that on one hand (53) it is a projection, $\operatorname{prox}_{\mathscr{K}}^{2}(\mathbf{x})=\operatorname{prox}_{\mathscr{K}}(\mathbf{x})$, and on the other hand there exists a linear subspace $\mathscr{L}$ containing $\mathbf{u}^{+}$such that this projection can be regarded as orthogonal. Since orthogonal projections are contractions, we have $\left\|\mathbf{u}^{+}\right\| \leq\left\|\mathbf{u}^{-}\right\|$. Moreover, because of the mutual orthogonality of the cones $\mathscr{T}_{\mathscr{C}}^{\frac{1}{(q}}(\mathbf{q})$ and $\mathscr{T}_{\mathscr{C}}(\mathbf{q})$, it can be shown [8]-[10], that $\mathbf{u}^{+}$from (53) is kinematical compatible, as soon as the trajectory approaches the boundary of the manifold from the admissible domain, $\mathbf{u}^{-} \in-\mathscr{T}_{\mathscr{C}}(\mathbf{q})$. This assures that (53) is the unique solution of the minimization problem (52) even in the case that all three conditions in (50) are considered as constraints.

In case of multi-contact impacts as discussed here, the concept of the Newtonian restitution coefficient from Sects. 2 and 4 can be abided and even consistently extended: In assigning the spheres of the fully inelastic and the fully elastic impact the values $\varepsilon=0$ and $\varepsilon=1$ and interpolating in between linearly, every $0 \leq \varepsilon \leq 1$ corresponds to a dissipation level, which itself corresponds to a sphere. However, the relative velocity that is changed during the impact according to (42), is still to be defined and is an open problem until now.

Recapitulating, the following can be stated: For multi-contact collision problems, the principle of maximum dissipation still gives a unique velocity $\mathbf{u}^{+}$after impact. This event is identified by the restitution coefficient zero. For all other restitution coefficients, the velocity after impact is restricted to a set by the conditions (50), but is still undefined. In order to be able to pick a particular element out of this set, we need an impact law in the sense of an additional, independent equation. The dynamics of the system can not be simply determined by choosing a certain dissipation level via the restitution coefficient as in single-contact collisions. A similar problem is the analytic extension of a geodesic on a manifold with an corner (Fig. 9). At a sharp bend, there exists still a unique reflecting hyperplane (Fig. 6). This property is lost at corners due to the singularity in curvature. Thus, the natural extension of the geodesic remains underdetermined, such as the post-impact velocity in the case of a completely elastic multicontact collision. 


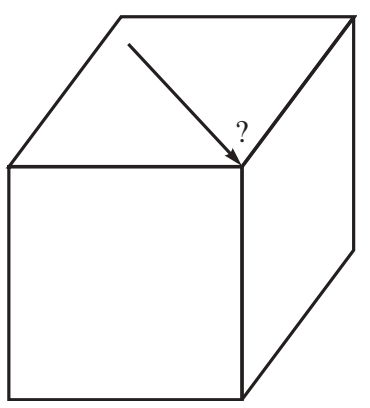

Fig. 9. On the natural extension problem of a geodesic at a corner

\section{Impacts with global dissipation index}

In this section we present one possible approach on how to single out one post-impact velocity $\mathbf{u}^{+}$for each energetic level from the admissible set $\mathscr{S}_{\mathbf{u}^{-}}(\mathbf{q})$. This approach requires the orthogonal decomposition of vectors with respect to an orthogonal pair of cones, which is a special case of Moreau's theorem, see, e.g. [7] for the full version and the proof:

Let $\left(\mathscr{R}, \mathscr{R}^{\perp}\right)$ be an orthogonal pair of closed convex cones in $\mathbb{R}^{f}$ that are mutually defined by

$\left(\mathscr{R}, \mathscr{R}^{\perp}\right)=\left\{\left(\mathbf{v}, \mathbf{v}^{\perp}\right) \mid \mathbf{v} \cdot \mathbf{v}^{\perp} \leq 0 \quad \forall \mathbf{v} \in \mathscr{R}, \forall \mathbf{v}^{\perp} \in \mathscr{R}^{\perp}\right\}$,

where $\cdot$ denotes the inner product on $\mathbb{R}^{f}$. Then

$\mathbf{u}=\mathbf{v}+\mathbf{v}^{\perp}, \quad \mathbf{v} \in \mathscr{R}, \quad \mathbf{v}^{\perp} \in \mathscr{R}^{\perp}, \quad \mathbf{v} \cdot \mathbf{v}^{\perp}=0$

holds for any $\mathbf{u} \in \mathbb{R}^{f}$ with unique elements $\mathbf{v} \in \mathbb{R}^{f}$ and $\mathbf{v}^{\perp} \in \mathbb{R}^{f}$.

We apply this decomposition on the pre-impact velocity $\mathbf{u}^{-}$with respect to the cones $\mathscr{T}_{\mathscr{C}}(\mathbf{q})$ and $\mathscr{T}_{\mathscr{C}}^{\perp}(\mathbf{q})$ that are orthogonal to each other by (48),

$\mathbf{u}^{-}=\mathbf{v}+\mathbf{v}^{\perp}, \quad \mathbf{v} \in \mathscr{T}_{\mathscr{C}}(\mathbf{q}), \quad \mathbf{v}^{\perp} \in \mathscr{T}_{\mathscr{C}}^{\perp}(\mathbf{q}), \quad \mathbf{v} \cdot \mathbf{v}^{\perp}=0$,

see Fig. 10. As members of the tangent cone and its orthogonal complement, $\mathbf{v}$ and $\mathbf{v}^{\perp}$ are called the tangential component and the normal component of the pre-impact velocity $\mathbf{u}^{-}$, respectively. We choose now the impact law that has been proposed in a similar form in [8] to determine the post-impact velocity $\mathbf{u}^{+}$: We leave the tangential component $\mathbf{v}$ unchanged by the impact, whereas the normal component $\mathbf{v}^{\perp}$ is "inverted" according to

$\mathbf{v}^{\times}:=-\varepsilon \mathbf{v}^{\perp} \quad(0 \leq \varepsilon \leq 1)$,

where $\varepsilon$ is the coefficient of restitution that addresses one of the energetic spheres from Sect. 5 . The post-impact velocity $\mathbf{u}^{+}$is then set to be

$\mathbf{u}^{+}:=\mathbf{v}+\mathbf{v}^{\times}$.

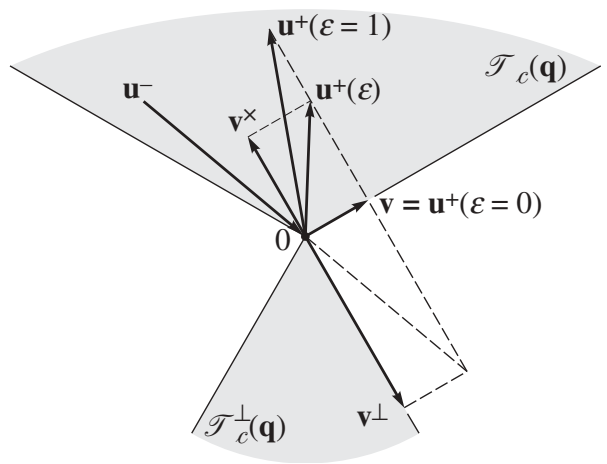

Fig. 10. On the construction of the impact law 
A collision event following the rules (56)-(58) is called an impact with global dissipation index.

From Fig. 10 one recognizes immediately a lot of properties of the impact law: It is always energetically consistent, because the kinetic energy $T$ satisfies $2 T^{+}=\left\|\mathbf{u}^{+}\right\|^{2} \leq\left\|\mathbf{u}^{-}\right\|^{2}=2 T^{-}$, where equality holds for $\varepsilon=1$ and maximal dissipation is achieved for $\varepsilon=0$. A completely elastic impact $(\varepsilon=1)$ can be interpreted as a reflection on a hyperplane $\mathscr{H}$ with normal $\mathbf{v}^{\perp}$, whereas a completely inelastic impact $(\varepsilon=0)$ corresponds to an orthogonal projection of $\mathbf{u}^{-}$on $\mathscr{H}$ to give $\mathbf{v}$. In terms of a minimization problem are $\mathbf{v}$ and $\mathbf{v}^{\perp}$ the nearest points to $\mathbf{u}^{-}$in the sets $\mathscr{T}_{\mathscr{C}}(\mathbf{q})$ and $\mathscr{T}_{\mathscr{C}}^{\perp}(\mathbf{q})$, respectively. The corresponding maps are called proximations and denoted by

$\mathbf{v}=\operatorname{prox}_{\mathscr{T}_{\mathscr{T}}(\mathbf{q})}\left(\mathbf{u}^{-}\right), \quad \mathbf{v}^{\perp}=\operatorname{prox}_{\mathscr{T}_{\frac{1}{8}}^{\perp}(\mathbf{q})}\left(\mathbf{u}^{-}\right)$.

For example, the impact law (56)-(58) might equivalently be stated in terms of proximations as

$\mathbf{u}^{+}=(1+\varepsilon) \operatorname{prox}_{\mathscr{T}_{\mathscr{\gamma}}(\mathbf{q})}\left(\mathbf{u}^{-}\right)-\varepsilon \mathbf{u}^{-}$

when the first equation in (59) is used. Further, we recognize that the proximation in (60) becomes the identity whenever $\mathbf{u}^{-} \in \mathscr{T}_{\mathscr{C}}(\mathbf{q})$. In this case $\mathbf{u}^{+} \equiv \mathbf{u}^{-}$, thus no impact occurs. An extension of this concept of global dissipation coefficient to moving boundaries or even to sets which are not tangentially regular can be found in [10] and [9], respectively.

The impact law (60) does not address every impact event possible, i.e., the whole set $\mathscr{S}_{\mathbf{u}^{-}}(\mathbf{q})$, but is restricted to a segment of the uniquely defined half-line of $\mathscr{T}_{\mathscr{C}}^{\frac{1}{\mathscr{C}}}(\mathbf{q})$ passing the origin and the point of maximal dissipation (53), see Fig. 11. The post-impact velocities (a) observed at Newton's cradle in the experiment, for example, can not be accessed by this impact law (60). It gives, instead, point (b) on the same energetic sphere $\varepsilon=1$, for which the first ball bounces back to the left after it hit the remaining four balls, which themselves move to the right as one single body of the same mass would do. If the impact law (60) is applied to systems which behave at the impact as dissipative as possible, such as the rocking rod (c), the right physical behavior is obtained.

In [10] a representation of the impact law (60) in local contact coordinates is derived. It has been shown that the local impact laws

$\left(\gamma_{i}^{+}+\varepsilon \gamma_{i}^{-}\right) \geq 0, \quad \Lambda_{i} \geq 0, \quad\left(\gamma_{i}^{+}+\varepsilon \gamma_{i}^{-}\right) \Lambda_{i}=0$

for each contact in the active set $\mathscr{H}$ (44) together with the impact equation (46) and the relative velocities (45) are equivalent to (60). The complementarity conditions in (61) express that each contact that takes an impulsive force $\Lambda_{i}>0$ is treated as in (42), but regarded as not to participate in the impact $\left(\Lambda_{i}=0\right)$ if the post-impact relative velocity fulfills $\gamma_{i}^{+}>-\varepsilon \gamma_{i}^{-}$, see also Fig. 12. The impact behavior (60) is called to have a global dissipation index, because the same restitution coefficient $\varepsilon$ is taken in (61) for each individual contact.

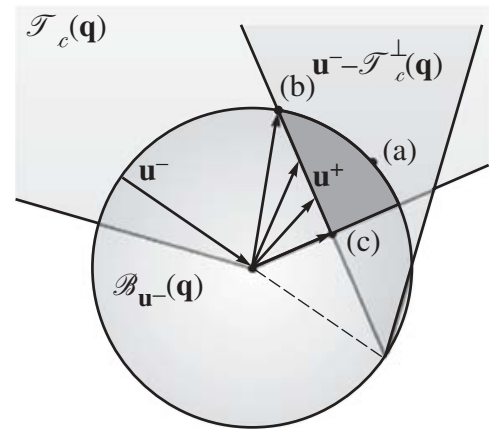

(a)

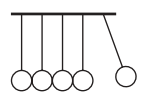

(b)

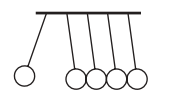

(c)

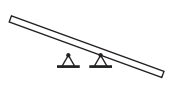

Fig. 11. Accessible post-impact velocity states 


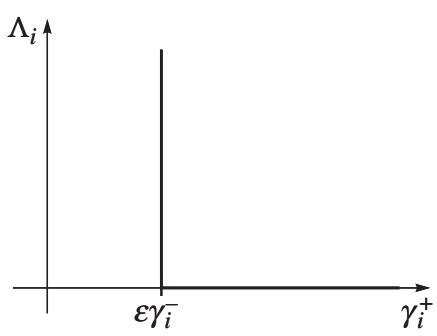

Fig. 12. Representation of the impact law in local coordinates

\section{Conclusion}

According to the results of Sect. 5, perfect multi-contact collisions cannot be solved by only using the dynamic, kinematic and energetic equations. Additional impact laws in the sense of independent equations must be provided to determine uniquely the velocity after the impact. The reason for this is a spatial discretization that is too rough such that too much physical information about the collision event is lost. This does not yet mean that such formulations for the numerical solution of dynamical systems have generally to be discarded, as it is often even inevitable to keep low-dimensional models because of memory size and calculation time. In this case, the missing information on how the impact behaves has to be taken from other sources, e.g., in advance by measurements on the real object or by refined models and improved calculations, but it must be assured that this information will find a well defined place in a theoretical framework for collisions. Such a theory, broad enough for a great class of collision types can be provided by a parameterization of the region $\mathscr{S}_{\mathbf{u}^{-}}(\mathbf{q})$ shown in Fig. 8 .

A related question concerns subsystem techniques. In this context, conditions have to be established under which impact laws on subsystems already identified can be further used if the subsystems collide with each other. This problem seems to have a solution for systems, at which the wave phenomena of each subsystem do not interfere with each other. Here, the demand for a more precise definition of the mechanical impact theory arises, in which wave effects are negligible by definition. In this context, the impacts with global dissipation index from Sect. 6 , containing the point of maximal dissipation, could play a crucial role if interpreted in combination with dispersion of waves.

Anyway, the principle of maximum dissipation seems to be the key to a deeper understanding of general impact theory in mechanics. The more dissipative the behavior of a system at the impact is, the closer the associated dissipation sphere to the point of maximum dissipation (Fig. 8), and the smaller the set on them from which possible post-impact velocities are taken. In the limit of maximum instantaneous dissipation, this region shrinks to a single point. Therewith, uncertainties in the choice of a impact law become less and less important. Once a discretization level deep enough has been found for which appearing collisions can be considered as fully inelastic, the impact can be resolved with great confidence by using the principle of maximum dissipation.

In addition to the Newton restitution coefficient $\varepsilon_{N}=-\gamma^{+} / \gamma^{-}$, one finds in literature also the Poisson restitution coefficient $\varepsilon_{P}=\Lambda^{(+)} / \Lambda^{(-)}$that relates the impulses during a compression and a decompression phase, and the energetic coefficient $\varepsilon_{E}$. For frictionless single-contact impact problems these concepts coincide. For perfect multi-contact collisions however, differences already occur, if the collision model from Fig. 4 together with a local impact law as in (42) is used on each contact. For example, the results after Newton and Poisson coincide, if $\left(\nabla g_{i} \cdot \nabla g_{j}\right) \varepsilon_{P j}=\left(\nabla g_{i} \cdot \nabla g_{j}\right) \varepsilon_{N i}$, thus if either all contacts affected by the impact do have the 
same restitution coefficient $\varepsilon_{P j}=\varepsilon_{N i}$, or if the contacts are decoupled in sense of the kinetic metric, $\nabla g_{i} \cdot \nabla g_{j}=0$. The question comes up, if generally local restitution coefficients may be used reasonably for perfect multi-contact impact problems, or if there is need to change to a new characterization of the impact using geometric invariant coefficients. It remains to be worked out, how to interpret these new coefficients in connection to the conventional restitution coefficients.

All investigations concerning impacts have been performed in this article by geometric methods based on the kinetic metric. Since mechanics has to be understood as metric-free, every result has to bee represented metric-free, what can be done by virtual work expressions and inclusions on the cotangent space and can be found in [10]. Further, all considerations are to be generalized to rheonomic systems, leading to moving sets $\mathscr{C}$. Basic steps on how to perform this task may be found in [10].

\section{Acknowledgement}

Parts of this work were obtained within the European project SICONOS IST-2001-37172 and supported by the Swiss Federal Office for Education and Science (BBW).

\section{References}

[1] Szabó, I.: Geschichte der mechanischen Prinzipien, 2nd ed. Basel: Birkhäuser 1979.

[2] Hagedorn, P.: Technische Mechanik, Bd. 3: Dynamik. Frankfurt, Thun: Verlag Harri Deutsch 1990.

[3] Glocker, Ch.: Dynamik von Starrkörpersystemen mit Reibung und Stössen. VDI-Fortschrittberichte Mechanik/Bruchmechanik, Reihe 18, Nr. 182, Düsseldorf: VDI-Verlag 1995.

[4] Pfeiffer, F., Glocker, Ch.: Multibody dynamics with unilateral contacts. New York: Wiley 1996.

[5] Ballard, P.: The dynamics of discrete mechanical systems with perfect unilateral constraints. Arch. Ratio. Mech. Anal. 154 (3), 199-274 (2000).

[6] Glocker, Ch.: Set-valued force laws: Dynamics of non-smooth systems. Berlin Heidelberg: Springer 2001.

[7] Rockafellar, R. T.: Convex analysis. Princeton, NJ: Princeton University Press 1972.

[8] Moreau, J. J.: Unilateral contact and dry friction in finite freedom dynamics. In: Non-smooth mechanics and applications. CISM Courses and Lectures 302 (Moreau, J.J., Panagiotopoulos, P.D., eds.), pp. 1-82. Wien: Springer 1988.

[9] Glocker, Ch.: Impacts with global dissipation index at reentrant corners. In: Proc. 3rd CMIS, Peniche, Portugal (Martins, J.A.C., Monteiro Marques, M., eds.), pp. 45-52. Dordrecht: Kluwer Academic Publishing 2002.

[10] Glocker, Ch.: The geometry of Newtonian impacts with global dissipation index for moving sets. In: Proc. Int. Conf. on Nonsmooth/Non-convex Mechanics, Thessaloniki, Greece (Baniotopoulos, C.C., ed.), pp. 283-290. Thessaloniki: Edition Ziti 2002.

Author's address: Ch. Glocker, IMES - Institute of Mechanical Systems, Swiss Federal Institute of Technology Zurich, ETH Zentrum, CH-8092 Zurich, Switzerland (E-mail: glocker@imes.mavt.ethz.ch) 